\title{
Supersolid order of frustrated hard-core bosons in a triangular lattice system
}

\author{
H. C. Jiang, ${ }^{1}$ M. Q. Weng, ${ }^{2}$ Z. Y. Weng, ${ }^{1}$ D. N. Sheng, ${ }^{3}$ and L. Balents ${ }^{4}$ \\ ${ }^{1}$ Center for Advanced Study, Tsinghua University, Beijing, 100084, China \\ ${ }^{2}$ Department of Physics, University of Science and Technology of China, Hefei 230026, China \\ ${ }^{3}$ Department of Physics and Astronomy, California State University, Northridge, California 91330, USA \\ ${ }^{4}$ Department of Physics, University of California, Santa Barbara, California 93106, USA
}

(Received 26 December 2008; published 30 January 2009)

\begin{abstract}
We numerically demonstrate that a supersolid phase exists in a frustrated hard-core boson system on a triangular lattice over a wide range of interaction strength. In the infinite repulsion (Ising) limit, we establish a mapping to the same problem with unfrustrated hopping, which connects the supersolid to the known results in that case. The weak superfluidity can be destroyed or strongly enhanced by a next-nearest-neighbor hopping term, which provides valuable information for experimental realization of a supersolid phase on optical lattice. A phase diagram for the frustrated anisotropic Heisenberg model on triangular lattice is also established.
\end{abstract}

DOI: 10.1103/PhysRevB.79.020409

PACS number(s): 75.10.Jm, 03.75.Lm, 05.30.Jp

\section{INTRODUCTION}

A supersolid phase is a state of matter exhibiting both diagonal and off-diagonal long-range order (ODLRO). ${ }^{1}$ Recent possible observation of a supersolid phase ${ }^{2}$ in ${ }^{4} \mathrm{He}$ under pressure has attracted a lot of interest. While the microscopic conditions under which clean ${ }^{4} \mathrm{He}$ could be in a supersolid phase are still unclear, supersolidity is established for hardcore bosons on a triangular lattice, which is the focus of many recent studies. ${ }^{3-7}$ The supersolid phase is an example of ordering by disorder demonstrated for hard-core boson system with unfrustrated nearest-neighbor $(\mathrm{NN})$ hopping and strong repulsion ${ }^{4-6}$ based on extensive quantum Monte Carlo simulations and theoretical analysis. These theoretical works are motivated in part by experimental realizations of lattice bosons in ultracold atom traps. ${ }^{8}$ Intriguingly, the superfluid density in the supersolid phase is found to be very small, possibly indicating that the system is near a phase boundary ${ }^{9}$ to an insulating phase. It is thus highly desirable to examine the stability of the supersolid phase in more extended models to determine the relevant perturbation and possibly to suggest a route of getting into a deep supersolid phase for experiment.

The nature of the state for the hard-core bosons with frustrated $\mathrm{NN}$ hopping on triangular lattice is another open issue, where the model can be mapped to the spin-1/2 XXZ antiferromagetic (AF) Heisenberg model which suffers from the sign problem. Historically this model was the first candidate proposed to realize a spin liquid ground state, ${ }^{10}$ although it turns out to still exhibit a three-sublattice AF long-rangeorder (LRO) in general, which may persist to large $J_{z}$ limit. ${ }^{11}$ However, extensive numerical studies have been limited to near the $\mathrm{SU}(2)$ point $^{12}$ and the precise nature of the ordering at larger $J_{z}$ (or the strong NN repulsion limit for the corresponding boson model) has not been well understood.

In this Rapid Communication, we present a systematic density-matrix renormalization-group (DMRG) and exact diagonalization (ED) numerical studies of the half-filled ground state of the frustrated model over a wide range of the $\mathrm{NN}$ repulsion. We show that a robust supersolid phase does exist in this model and it can be related to the well-known supersolid phase of an unfrustrated NN hopping model in infinite repulsion (Ising) limit, where a precise mapping between the two models by a sign transformation can be established. Furthermore, we reveal that the supersolid phase is close to a transition to an insulating phase, and correspondingly the superfluidity can be strongly enhanced (or easily switched off) by tuning a next-nearest-neighbor (NNN) hopping term. Our results have also provided a theoretical understanding of the nature of the ground state of the frustrated Heisenberg model on triangular lattice at the large $J_{z}$ limit.

\section{SUPERSOLID PHASE FROM ISOTROPIC SU(2) POINT TO LARGE $J_{z}$ LIMIT}

We consider a simple model of hard-core bosons at halffilling on the triangular lattice interacting via a repulsive term,

$$
H=-t \sum_{\langle i j\rangle}\left(b_{i}^{\dagger} b_{j}+b_{j}^{\dagger} b_{i}\right)+\sum_{\langle i j\rangle} V\left(n_{i}-\frac{1}{2}\right)\left(n_{j}-\frac{1}{2}\right),
$$

where $b_{i}^{\dagger}$ is a boson creation operator and $n_{i}$ is a boson number operator. $\langle i j\rangle$ denotes $\mathrm{NN}$ sites and we shall mainly consider the frustrated boson hopping at $t<0$. This boson Hamiltonian is equivalent to-by a standard mapping from hard-core bosons to $S=1 / 2$ spins-an $X X Z$ Heisenberg model on the triangular lattice,

$$
\mathcal{H}=\sum_{\langle i j\rangle}\left[\frac{J_{\perp}}{2}\left(S_{i}^{\dagger} S_{j}^{-}+\text {H.c. }\right)+J_{z} S_{i}^{z} S_{j}^{z}\right],
$$

with $J_{z}=V$ and $J_{\perp}=-2 t$. In the spin language, a chargedensity wave (solid) order implies infinite-range correlations of the $z$ component of spins at a nonzero wave vector, while the superfluid order is equivalent to the in-plane ordering of the spins also at a nonzero wave vector for the frustrated system. In the following analysis, we will work interchangeably in terms of bosons and spin variables.

We first present the numerical results based on DMRG method $^{13}$ for a triangular lattice with the total number of sites $N=N_{1} \times N_{2}$. We keep up to $m=4096$ states in each DMRG block for most systems and the truncation error is of the 

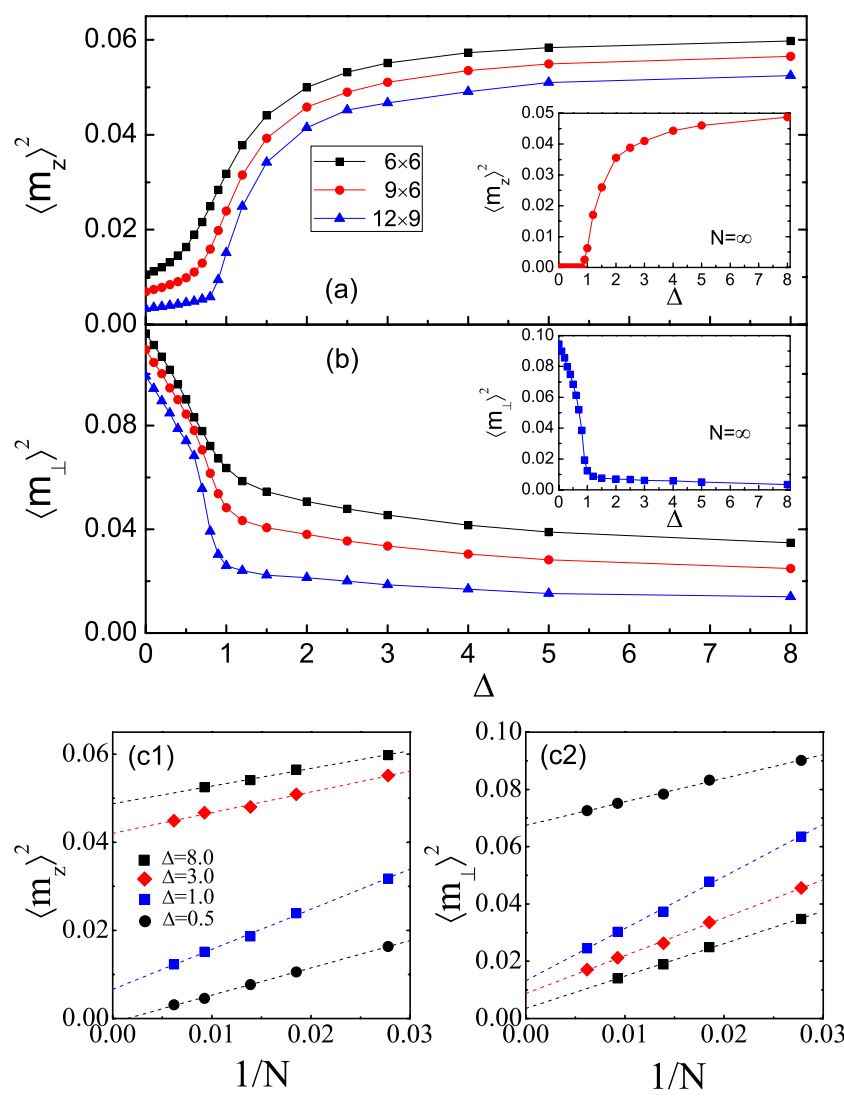

FIG. 1. (Color online) The order-parameter squared, $\left\langle m_{z}\right\rangle^{2}$ and $\left\langle m_{\perp}\right\rangle^{2}$, as functions of the anisotropy $\Delta=J_{z} / J_{\perp}$ are shown in (a) and (b), respectively, with $N=6 \times 6,9 \times 6$, and $12 \times 9$. The insets are the corresponding extrapolations in the thermodynamic limit. Examples of finite-size scaling of the order parameters are also shown in (c1) and (c2) with system size up to $N=9 \times 18$.

order or less than $10^{-5}$. We make use of the periodic boundary condition to reduce the finite-size effect for a more reliable extrapolation to the thermodynamic limit. To analyze the magnetic properties of the system, we calculate the structure factors $S_{z}(\mathbf{q})$ and $S_{\perp}(\mathbf{q})$ defined by $S_{z}(\mathbf{q})$ $=\frac{1}{N} \sum_{i, j} e^{-i \mathbf{q}\left(\mathbf{r}_{i}-\mathbf{r}_{j}\right)}\left\langle S_{i}^{z} S_{j}^{z}\right\rangle$ and $S_{\perp}(\mathbf{q})=\frac{1}{N} \sum_{i, j} e^{-i \mathbf{q}\left(\mathbf{r}_{i}-\mathbf{r}_{j}\right)}\left\langle S_{i}^{+} S_{j}^{-}\right\rangle$.

The obtained $S_{z}(\mathbf{q})$ and $S_{\perp}(\mathbf{q})$ show Bragg peaks at the corners of the hexagonal Brillouin zone [e.g., at $q_{0}$ $=( \pm 4 \pi / 3,0)]$. In particular, at small $\Delta \equiv J_{z} / J_{\perp}<1$, the peak of $S_{z}\left(\mathbf{q}_{0}\right)$ is very weak, while that of $S_{\perp}\left(\mathbf{q}_{0}\right)$ is very sharp, representing the dominant $\mathrm{AF}$ correlation in the $X Y$ plane. With the increase of $\Delta, S_{z}\left(\mathbf{q}_{0}\right)$ grows continuously and its value becomes bigger than the in-plane ones passing the point $\Delta=1$. One can obtain the magnetic order parameters based on the finite-size scaling of the peak values of $S_{z}\left(\mathbf{q}_{0}\right)$ and $S_{\perp}\left(\mathbf{q}_{0}\right)$. Specifically, the average magnetization $\left\langle m_{z}\right\rangle$ and $\left\langle m_{\perp}\right\rangle$ can be determined by $\left\langle m_{z}\right\rangle^{2}=S_{z}\left(\mathbf{q}_{0}\right) / N$ and $\left\langle m_{\perp}\right\rangle^{2}$ $=S_{\perp}\left(\mathbf{q}_{0}\right) / N$, which are shown vs $\Delta$ at $N=36,54$, and 108 in the main panel of Figs. 1(a) and 1(b).

Nonzero $\left\langle m_{z}\right\rangle^{2}$ and $\left\langle m_{\perp}\right\rangle^{2}$ in the thermodynamic limit will correspond to the diagonal LRO and ODLRO, respectively. Examples of the finite-size scaling are shown in Figs. 1(c1) and (c2) by plotting $\left\langle m_{z}\right\rangle^{2}$ and $\left\langle m_{\perp}\right\rangle^{2}$ as functions of $1 / N$. Thus obtained order parameters extrapolated to the thermodynamic limit are presented in the insets of Figs. 1(a) and

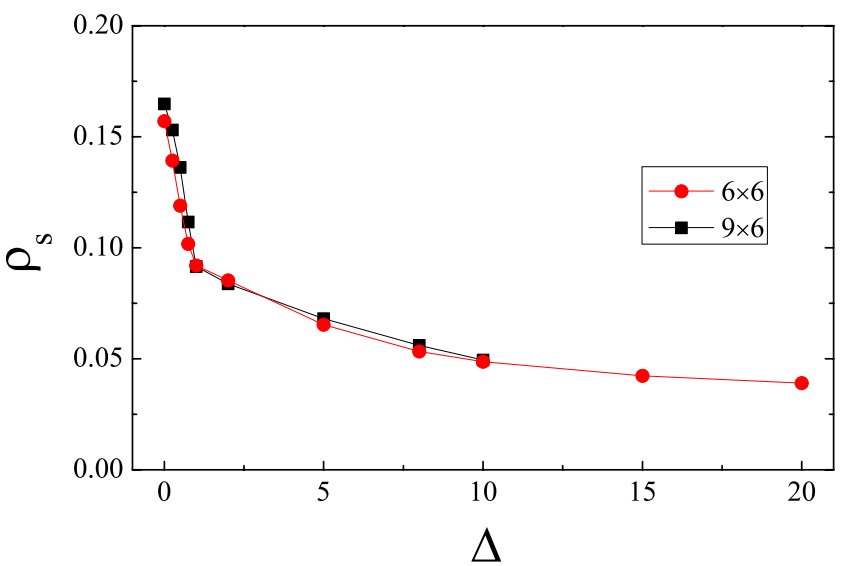

FIG. 2. (Color online) Superfluid stiffness $\rho_{S}$ (in units of $J_{\perp}$ ) as a function of the anisotropy $\Delta=J_{z} / J_{\perp}$ obtained from $\operatorname{ED}(N=36)$ or DMRG $(N=54)$ calculations.

1(b). At small $\Delta$, the system is in the pure superfluid phase with magnetic order solely lying in the $X Y$ plane with $\left\langle m_{\perp}\right\rangle^{2} \neq 0$ and $\left\langle m_{z}\right\rangle=0$ (cf. the inset of Fig. 1). By contrast, at $\Delta>1$ the three-sublattice antiferromagetic long-range order develops in both $z$ direction and $X Y$ plane characterized by nonzero values of $\left\langle m_{z}\right\rangle^{2}$ and $\left\langle m_{\perp}\right\rangle^{2}$. Here $\left\langle m_{z}\right\rangle$ monotonically increases with $\Delta$ from the isotropic point $(\Delta=1)$ consistent with the spin-wave picture of coplanar ordering in the $X Z$ plane. For the corresponding boson system, our results suggest a supersolid phase with coexisting diagonal LRO and ODLRO at $\Delta \geq \Delta_{c}$. The phase boundary $\Delta_{c}$ between the superfluid phase and supersolid phase is very close to the isotropic point $\Delta_{c}=\left(J_{z} / J_{\perp}\right)_{c}=(V / 2|t|)_{c} \sim 1.00$.

We note that at large $J_{z}$ case, e.g., $\Delta=8,\left\langle m_{z}\right\rangle^{2}=0.049$ $\left(\left\langle m_{z}\right\rangle=0.24\right)$ which is much larger than the corresponding value at the isotropic point, while the $X Y$-plane magnetization reduces to $\left\langle m_{\perp}\right\rangle^{2}=0.0036\left(\left\langle m_{\perp}\right\rangle=0.06\right)$ in the thermodynamic limit. Though this superfluid ordering is small, its value actually is comparable with that of the unfrustrated hard-boson supersolid ${ }^{4-6}$ at the same large $J_{z}$ limit as we have checked numerically. In the following, we can further establish the presence of the superfluidity through the calculation of the superfluid density $\rho_{s}$ by adding a nonzero twist phase at the system boundary with $\rho_{s}=\frac{\partial^{2} E}{\partial \theta_{x}^{2}} \propto\left[E\left(\theta_{x}=\pi\right)\right.$ $\left.-E\left(\theta_{x}=0\right)\right]$. We obtain $\rho_{s}$ by adding a twist phase $\theta_{x}=\pi$ in both ED and DMRG calculations, ${ }^{14}$ which are shown in Fig. 2 as a function of $\Delta$ for $N=36$ and $N=54$. From the figure, we can see that the finite-size effect for $\rho_{s}$ is very weak and $\rho_{s}$ should remain finite in the thermodynamic limit consistent with the finite $\left\langle m_{\perp}\right\rangle^{2}$ in Fig. 1. At $J_{z}>10$, the DMRG becomes difficult to converge as the energy from $J_{z}$ term becomes dominant; the ED results further suggest that the obtained $\rho_{s}$ should be nonzero over the whole range of $J_{z}$ with a value matching with the ones for the unfrustrated bosons in the large $J_{z}$ limit. $^{6}$

\section{SUPERSOLID ORDER IN THE ISING LIMIT}

Now we turn to the interesting limit of $\Delta \rightarrow \infty$, where the direct in-plane magnetic ordering in the numerical results is 
very weak and the spin-wave theory suggests that it vanishes as square root of $J_{\perp} / J_{z}$. Clearly, here a rigorous examination is desired. At $J_{z} \rightarrow \infty$, the $X X Z$ model in Eq. (2) reduces to the classical Ising AF on a triangular lattice. This classical model is well known to have a macroscopic degeneracy of ground states, which correspond to all spin configurations with exactly one frustrated bond per triangle. ${ }^{15}$ In this limit, the $X X Z$ model reduces to

$$
H_{\infty}=J_{\perp} / 2 \sum_{\langle i j\rangle} \hat{P}_{C}\left(S_{i}^{+} S_{j}^{-}+\text {H.c. }\right) \hat{P}_{C},
$$

where $\hat{P}_{C}$ is a projection operator onto the classical Ising ground-state manifold.

The unfrustrated model with $J_{\perp}<0$ has been studied previously and shown to exhibit supersolid order. ${ }^{4-6}$ We now show how supersolidity in the frustrated case $J_{\perp}>0$ can be deduced from those known results. We first consider thermodynamic and other properties that can be deduced from the partition function and diagonal expectation values of the form,

$$
Z\left(\mathcal{O} ; J_{\perp}\right)=\operatorname{Tr}\left\{\hat{P}_{C} \mathcal{O}\left[\left(S_{i}^{z}\right)\right] e^{-\beta H_{\infty}}\right\},
$$

where $\mathcal{O}$ is any function of the $z$ components of the spins (including the identity operator where $Z$ reduces to the partition function). From such quantities, we can calculate the free energy and the diagonal (solid) correlations. We show that $Z$ is an even function of $J_{\perp}$, and so these properties are identical for the frustrated and unfrustrated cases. To see this, consider the high-temperature expansion of $Z$ in powers of $\beta J_{\perp}$. The terms in the expansion consist of successive actions of bond operators of the form $\hat{P}_{C} S_{i}^{+} S_{j}^{-} \hat{P}_{C}$ on nearestneighbor links, with a factor of $\beta J_{\perp}$ accompanying each bond operator. To achieve a nonzero expectation value in the trace, the boson number on each site must be unchanged after the action of all these operators. Graphically, we may represent each factor of $S_{i}^{+} S_{j}^{-}$on the lattice as an arrow pointing from site $j$ to site $i$, and we require this "vector field" to have zero divergence, i.e., the arrows close into "exchange" loops. Now consider the contribution from any particular state in the trace. Due to the projection, each bond operator has a nonzero action only if $i$ and $j$ are "flippable," i.e., the two other spins on each triangle containing $i$ or $j$ are antiparallel. Now let us circumscribe each exchange loop on our graphical representation by a neighboring loop as in Fig. 3. In order that all sites on the exchange loops are flippable, spins on the neighboring loops must alternate, which requires that all of the neighboring loops must have an even number of sites. This in turn requires that the total number of links on each exchange loop is even. Thus $Z\left[\mathcal{O} ; J_{\perp}\right]$ is indeed an even function of $J_{\perp}$.

Now consider the off-diagonal correlation function,

$$
\left\langle S_{j}^{+} S_{i}^{-}\right\rangle=Z^{-1} \operatorname{Tr}\left[\hat{P}_{C} S_{j}^{+} S_{i}^{-} e^{-\beta H_{\infty}}\right] .
$$

Once again, one may consider the high-temperature expansion of the numerator (the denominator $Z$ has already been shown to be even). In this case, contributions must be divergenceless except at the sites $i$ and $j$, which appear as source and sink, respectively. One can understand the behavior by

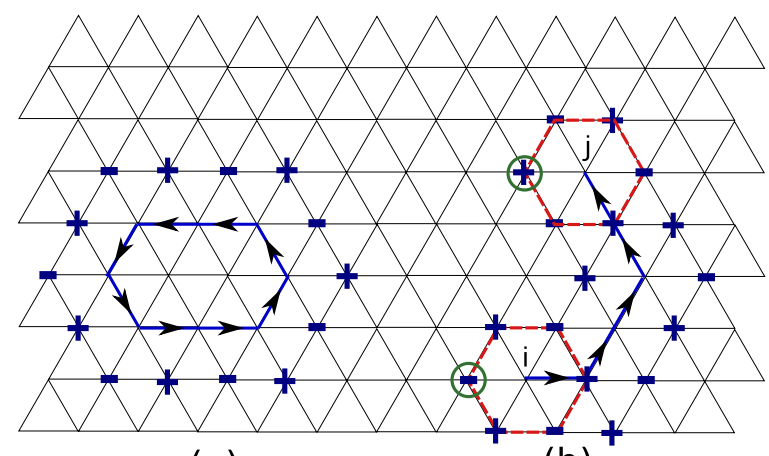

(a)

(b)

FIG. 3. (Color online) Illustration of the paths contributing to the high-temperature expansion and the associated sign rule. In (a), we show a representative loop appearing in the expansion of the partition function. The \pm signs show one of two alternating spin configurations allowed around the loop, which allows only evenlength loops. In (b), we show a representative path in the expansion of the off-diagonal correlation function between sites $i$ and $j$. For such a path with an odd number of steps, the auxiliary sites $i$ $+\hat{a}, j+\hat{a}$ (shown in circles) must be antiparallel.

considering just the simplest terms, in which the arrows form a single path connecting $i$ to $j$ (see Fig. 3). Now form a tightly circumscribing loop about this path. As above, for any state in the trace to contribute, the spins $S_{k}^{z}$ on the sites of this neighboring loop must alternate. Moreover, the six spins neighboring $i$ and $j$ must also alternate since $S_{j}^{+} S_{i}^{-}$acts directly on these states. Now consider the product $4 S_{i+\hat{a}}^{z} S_{j+\hat{a}}^{z}$, where $\hat{a}$ is any nearest-neighbor vector, acting on a state which contributes to the trace. Because of the alternating spins around the circumscribing loop, this factor gives the parity of the exchange path, i.e., it equals +1 for an even path and -1 for an odd path. One may show that this conclusion is unaffected by additional closed loops, which appear as higher-order terms in the high-temperature series. Since this is true for every term in the expansion, we find $\left.\left\langle S_{j}^{+} S_{i}^{-}\right\rangle\right|_{J_{\perp}}>0$ $=\left.\left\langle 4 S_{i+\hat{a}}^{z} S_{j+\hat{a}}^{z} S_{j}^{+} S_{i}^{-}\right\rangle\right|_{-J_{\perp}}$.

The above observations lead us to the conclusion that the supersolid phase survives even for the frustrated hard-core boson system at strong repulsion $\left(V=J_{z} \rightarrow \infty\right)$ limit as it maps to the unfrustrated model. ${ }^{4-6}$

\section{ENHANCEMENT OF THE SUPERFLUIDITY AND THE ORDERING OF THE SUPERSOLID PHASES}

To understand the underlying reason why the superfluid stiffness is relatively weak, ${ }^{9}$ we add a NNN hopping $t^{\prime}=$ $-J^{\prime} / 2$ term. For simplicity we only present the results for $J_{\perp}=-2 t>0$ and large $J_{z}$ (Ising) limit, although the obtained results also apply to both models with a finite range of $J_{z}$.

The superfluid stiffness is calculated using the ED method for the projected Hamiltonian. As illustrated in Fig. $4, \rho_{s}$ is relatively small at $J^{\prime}=0$ compared to its value in the region $J^{\prime} / J_{\perp}<0$ (negative sign represents an unfrustrated NNN hopping). In fact, $\rho_{s}$ monotonically increases with a negative $J^{\prime}$ and when $J^{\prime}=-J_{\perp}, \rho_{s}$ becomes comparable to the value of a pure superfluid phase (i.e., the ferromagnetic $X Y$ model on 


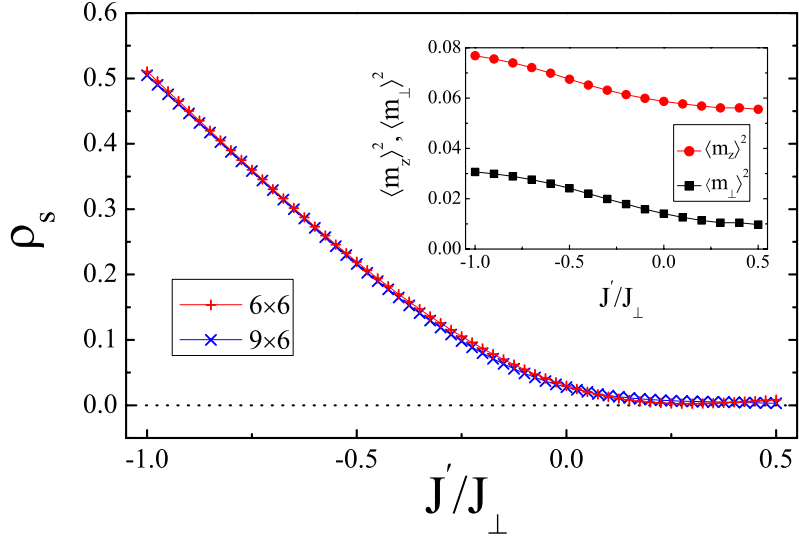

FIG. 4. (Color online) Superfluid stiffness $\rho_{s}$ (in units of $J_{\perp}$ ) vs $J^{\prime} / J_{\perp}$ for systems with NNN hopping $t^{\prime}$ (superexchange $J^{\prime}$ ). The finite-size order parameters $\left\langle m_{z}\right\rangle^{2}$ and $\left\langle m_{\perp}\right\rangle^{2}$ at $N=54$ are shown in the inset.

triangular lattice $\left.{ }^{6}\right)$. Clearly a boson system at $J^{\prime}=0$ is indeed near the phase boundary of an insulating phase, which occurs at $\left(J^{\prime} / J_{\perp}\right)_{c} \sim 0.2$ (which we further identify as a solid phase with diagonal LRO). The finite-size order parameters $\left\langle m_{z}\right\rangle^{2}$ and $\left\langle m_{\perp}\right\rangle^{2}$ for $N=54$ are also shown in the inset of Fig. 4, where the enhancement of the peaks of the structure factors $S_{z}\left(q_{0}\right)$ and $S_{\perp}\left(q_{0}\right)$ are clearly seen as we continuously turn on the negative $J^{\prime}$. Thus the resulting phase is a supersolid phase with strong diagonal LRO and superfluidity.

These observations and the precise nature of the supersolid ordering can be rationalized by simple energetic arguments in the large $J_{z}$ limit. For the NN hopping case $\left(J^{\prime}\right.$ $=0)$, the constraint that neighboring spins to the hopping path must alternate tends to enhance hopping that takes " $60^{\circ}$ " turns (forming hexagonal path), which keeps the bosons on two of the three sublattices. Moreover, the third sublattice on which hopping does not proceed must be substantially polarized. Thus the three-sublattice ordering $\left\langle S_{z}\right\rangle=(-m,-m, 2 m$ $+\delta$ ) is favored energetically consistent with a ferrimagnetic- ordered phase. ${ }^{7,16}$ When the NNN hopping term is dominant $\left(-J^{\prime} \geq J_{\perp}\right)$, bosons tend to hop on a single (say A) sublattice, while spins on the neighboring sites from sublattices B and $\mathrm{C}$ are individually preferred to be ferromagnetically aligned with $\mathrm{B}$ and $\mathrm{C}$ spins antiparallel to each other. This corresponds to $\left\langle S_{z}\right\rangle=(0, m,-m)$ or "antiferromagnetic" ordering, which we therefore expect in the large $-J$ ' limit. This is indeed supported by numerics, which will be presented elsewhere.

In summary, we have established a robust supersolid phase for the frustrated hard-core bosons on a triangular lattice at half-filling based on extensive numerical calculations and analytical analysis. The observed supersolidity is an example of ordering by disorder elegantly realized for such a frustrated system. Furthermore, we have found that the supersolid phases for the hard-core boson models with only NN hoppings are quite close to a pure solid phase in both frustrated and unfrustrated cases. But a small unfrustrated NNN hopping term can push the boson systems into a deep supersolid phase with greatly enhanced superfluidity. Our theoretical study can thus provide a solid foundation for the experimental realization of supersolid phase on a triangular optical lattice.

\section{ACKNOWLEDGMENT}

The authors would like to thank R. G. Melko for insightful discussions. This work is supported by the NSFC under Grants No. 10688401 (H.C.J. and Z.Y.W.) and No. 10804103 (M.Q.W.), the National Program for Basic Research of MOST, China (Z.Y.W.), the DOE under Grant No. DEFG02-06ER46305, the NSF under Grants No. DMR0605696, No. DMR-0611562 (H.C.J. and D.N.S.), and No. DMR-0804564 (L.B.), and the Packard Foundation (L.B.). In finishing this work, we became aware of a parallel work ${ }^{16}$ which has reached similar conclusion based on different approaches. We are grateful to the author (A. V.) for sending us the preprint before submission.
${ }^{1}$ A. F. Andreev and I. M. Lifshitz, Sov. Phys. JETP 29, 1107 (1969); G. Chester, Phys. Rev. A 2, 256 (1970).

${ }^{2}$ E. Kim and M. H. W. Chan, Nature (London) 427, 225 (2004); Science 305, 1941 (2004).

${ }^{3}$ R. Moessner and S. L. Sondhi, Phys. Rev. B 63, 224401 (2001).

${ }^{4}$ S. Wessel and M. Troyer, Phys. Rev. Lett. 95, 127205 (2005).

${ }^{5}$ D. Heidarian and K. Damle, Phys. Rev. Lett. 95, 127206 (2005).

${ }^{6}$ R. G. Melko, A. Paramekanti, A. A. Burkov, A. Vishwanath, D. N. Sheng, and L. Balents, Phys. Rev. Lett. 95, 127207 (2005).

${ }^{7}$ Arnab Sen, P. Dutt, K. Damle, and R. Moessner, Phys. Rev. Lett. 100, 097202 (2008).

${ }^{8}$ I. Bloch, J. Dalibard, and W. Zwerger, Rev. Mod. Phys. 80, 885 (2008).
${ }^{9}$ A. A. Burkov and L. Balents, Phys. Rev. B 72, 134502 (2005).

${ }^{10}$ P. Fazekas and P. W. Anderson, Philos. Mag. 30, 423 (1974).

${ }^{11}$ N. Read and S. Sachdev, Phys. Rev. B 42, 4568 (1990).

${ }^{12}$ B. Bernu, P. Lecheminant, C. Lhuillier, and L. Pierre, Phys. Rev. B 50, 10048 (1994); L. Capriotti, A. E. Trumper, and S. Sorella, Phys. Rev. Lett. 82, 3899 (1999).

${ }^{13}$ S. R. White, Phys. Rev. B 72, 180403(R) (2005).

${ }^{14}$ D. N. Sheng, O. I. Motrunich, S. Trebst, E. Gull, and M. P. A. Fisher, Phys. Rev. B 78, 054520 (2008).

${ }^{15}$ J. Stephenson, J. Math. Phys. 11, 413 (1970).

${ }^{16}$ F. Wang, F. Pollmann, and A. Vishwanath, Phys. Rev. Lett. 102, 017203 (2009). 